\title{
RECURSIVE EQUIVALENCE TYPES AND COMBINATORIAL FUNCTIONS ${ }^{1}$
}

\author{
BY J. MYHILL
}

Communicated by P. C. Rosenbloom, August 23, 1958

Introduction. Recursive equivalence types and isols. The theory of recursive equivalence types (R.E.T.s; see $[1 ; 2 ; 3 ; 4]$ ) is a constructive counterpart of Cantor's theory of transfinite numbers. Two sets $\alpha$ and $\beta$ of nonnegative integers are called recursively equivalent if one can be mapped onto the other by a one-one partial recursive function; we write $\alpha \simeq \beta$. The equivalence classes into which the class of all sets of nonnegative integers is decomposed by this equivalence relation are called recursive equivalence types; the R.E.T. to which a set $\alpha$ belongs will be denoted by Req $\alpha$. The elementary arithmetic operations on R.E.T.s are defined by

$$
\begin{aligned}
& \operatorname{Req} \alpha+\operatorname{Req} \beta=\operatorname{Req}(\{2 n \mid n \in \alpha\}+\{2 n+1 \mid n \in \beta\}), \\
& \operatorname{Req} \alpha \cdot \operatorname{Req} \beta=\operatorname{Req}\left\{2^{m} \cdot 3^{n} \mid m \in \alpha \& n \in \beta\right\} .
\end{aligned}
$$

It is easy to establish the existence and uniqueness of sums and products so defined, and to prove the formulas $(A+B)+C=A+(B+C)$, $A+B=B+A, \quad(A B) C=A(B C), \quad A B=B A, \quad A(B+C)=A B+A C$, $A+0=A, A B=0 \leftrightarrow(A=0$ or $B=0)$, where 0 is the R.E.T. of the empty set. Two finite sets are recursively equivalent if and only if they have the same number of elements; thus it is permissible to identify the R.E.T.s of finite sets with the nonnegative integers. The R.E.T.s are partially ordered by the relation $A \leqq B$ which holds when $A+C=B$ for some R.E.T. $C$.

Amongst R.E.T.s a special role is played by those types $A$ for which $A \neq A+1$; these types are called isols and the sets they characterize, isolated sets. Isolated sets are the constructive analogues of sets which are finite in the sense of Dedekind; they are precisely those sets which contain no infinite recursively enumerable subset. The isols are a proper subcollection of the R.E.T.s, and the nonnegative integers are a proper subcollection of the isols.

Arithmetical formulas of certain forms hold automatically for isols (and sometimes for R.E.T.s generally) provided they hold for nonnegative integers. So far $([2 ; 4])$ this has only been observed for formulas involving addition, an exponentiation multiplication. The

\footnotetext{
${ }^{1}$ The research reported in this note was done while the writer received support from the Institute for Advanced Study, and from NSF grant G-3466.
} 
purpose of this note is to announce similar results for formulas involving a wider class of functions.

1. Combinatorial mappings and combinatorial functions of nonnegative integers. Let $V$ be the class of all sets of nonnegative integers. A mapping

$$
\phi: V \rightarrow V
$$

is called a combinatorial mapping if the following conditions are satisfied: (I) if $\alpha$ is finite, so is $\phi(\alpha)$ : (II) the cardinality of $\phi(\alpha)$ is determined by that of $\alpha$ : (III) $\phi$ possesses a quasi-inverse $\phi^{-1}$ such that for any $x \in \mathrm{U}_{\alpha \in V} \phi(\alpha)$ we have

$$
x \in \phi(\beta) \leftrightarrow \phi^{-1}(x) \subset \beta .
$$

In virtue of (I)-(II), $\phi$ induces a number-theoretic function $f_{\phi}$ such that if $\alpha$ has $n$ elements, $\phi(\alpha)$ has $f_{\phi}(n)$ elements. Such a function $f_{\phi}$ is called a combinatorial function. The functions $x^{k}, k^{x}, C_{x, k}, x !, x^{x}$ are combinatorial; and if $f(x)$ and $g(x)$ are combinatorial, so are $f g(x)$, $f(x)+g(x)$ and $f(x) \cdot g(x)$.

Every number-theoretic function can be expressed uniquely in the form

$$
f(x)=\sum_{i} c_{i} C_{x, i}
$$

where the $c_{i}$ are integers. $f$ is combinatorial if and only if (1) holds with all $c_{i} \geqq 0$.

2. Combinatorial functions of R.E.T.s. The combinatorial function (1) is induced by the combinatorial mapping

$$
\phi(\alpha)=\left\{2^{m} \cdot 3^{n} \mid \rho_{m} \subset \alpha \& n<c_{r(m)}\right\}
$$

where the $c_{i}$ are the same as in (1), $\left\{\rho_{i}\right\}$ is the canonical enumeration of finite sets (see [5]) and $r(i)$ is the number of elements of $\rho_{i}$. A combinatorial mapping $\phi$ satisfying (2) for certain constants $c_{i}$ is called a normal combinatorial mapping. For normal $\phi$ (even when $c_{i}$ is not a recursive function of $i), \alpha \simeq \beta$ implies $\phi(\alpha) \simeq \phi(\beta)$. Hence every combinatorial function $f$ of nonnegative integers can be canonically extended to a function $F$ of R.E.T.s by setting

$$
F(\operatorname{Req} \alpha)=\operatorname{Req} \phi(\alpha)
$$

where $\phi$ is the unique normal combinatorial mapping which induces $f$. If $f$ is recursive, the canonical extension of $f$ to R.E.T.s is called a recursive combinatorial (r.c.) function of R.E.T.s. The functions $A^{k}$, $k^{A}, C_{A, k}, A$ !, $A^{A}$ are r.c. functions; their definitions as canonical ex- 
tensions of the corresponding number-theoretic functions are equivalent to the definitions used in $[1 ; 3 ; 4]$. The composition, sum and product of two r.c. functions are r.c.

\section{Theorems.}

T1. Let $F$ be r.c. Then $A \leqq B$ implies $F(A) \leqq F(B)$. Moreover, if $F$ is not a constant and $A$ and $B$ are isols exceeding a certain finite number depending on $F, A \neq B$ implies $F(A) \neq F(B)$. In particular if $A, B$ are isols we have $A^{k}=B^{k} \rightarrow A=B$ for $A, B, k \geqq 1 ; k^{A}=k^{B} \rightarrow A$ $=B$ for $k \geqq 2 ; C_{A, k}=C_{B, k} \rightarrow A=B$ for $A, B \geqq k \geqq 1 ; A !=B ! \rightarrow A=B$ and $A^{A}=B^{B} \rightarrow A=B$ for $A, B \geqq 1$.

T2. Let $F$ be r.c. Then if $A$ is an isol so is $F(A)$; the converse is true provided that $F$ is not a constant.

T3. Let $F$ and $G$ be r.c. Then

(a) If $F(n)=G(n)$ for infinitely many finite $n$, then $F(A)=G(A)$ for at least one infinite isol $A$.

(b) If $F(n)=G(n)$ for all but finitely many finite $n$, then $F(A)$ $=G(A)$ for all infinite R.E.T.s $A$.

(c) If $F(n) \neq G(n)$ for infinitely many finite $n$, then $F(A) \neq G(A)$ for at least one infinite isol $A$.

(d) If $F(n) \neq G(n)$ for all but finitely many finite $n$, then $F(A)$ $\neq G(A)$ for all infinite isols $A$ (but in general not for all infinite R.E.T.s $A$; take $F(n)=n, G(n)=n+1)$.

4. Generalizations. A combinatorial function of $k$ nonnegative integer arguments $x_{1}, \cdots, x_{k}$ is a function

$$
f\left(x_{1}, \cdots, x_{k}\right)=\sum_{a_{1}, \cdots, a_{k}}\left[c_{a_{1} \cdots a_{k}} \prod_{i=1}^{n} C_{x_{i}, a_{i}}\right]
$$

with all the $c_{a_{1} \ldots a_{k}}$ nonnegative. $x y, x+y$ and $x^{y}$ (but not $C_{x, y}$ ) are combinatorial. The family of all combinatorial functions is closed under composition. The canonical extension to R.E.T.s of combinatorial functions of several arguments, and the notion of an r.c. function of several arguments, are defined in a manner exactly parallel to the one-argument case. Analogues of T2 and of all parts of T3 can then be proved. In particular if $F$ and $G$ are r.c. functions of $k$ arguments, we have

T3*(b). If $F\left(x_{1}, \cdots, x_{k}\right)=G\left(x_{1}, \cdots, x_{k}\right)$ for all finite $x_{1}, \cdots, x_{k}$, then $F\left(A_{1}, \cdots, A_{k}\right)=G\left(A_{1}, \cdots, A_{k}\right)$ for all infinite R.E.T.s $A_{1}, \cdots, A_{k}$.

T3*(d). If $F\left(x_{1}, \cdots, x_{k}\right) \neq G\left(x_{1}, \cdots, x_{k}\right)$ for all but finitely many $k$-tuples $\left(x_{1}, \cdots, x_{k}\right)$ of nonnegative integers, then $F\left(A_{1}, \cdots, A_{k}\right) \neq G\left(A_{1}, \cdots, A_{k}\right)$ for all infinite isols $A_{1}, \cdots, A_{k}$. 
Notice that $\mathrm{T} 3 *(\mathrm{~d})$ contains the principal result of [2] as a special case.

A. Nerode has obtained generalizations of $\mathrm{T} 3$ in a different direction. For example, he has shown that if $F, G, H, L$ are r.c. functions such that for all finite $x_{1}, \cdots, x_{k}$

$$
F\left(x_{1}, \cdots, x_{k}\right)=G\left(x_{1}, \cdots, x_{k}\right) \rightarrow H\left(x_{1}, \cdots, x_{k}\right)=L\left(x_{1}, \cdots, x_{k}\right)
$$

then for all infinite isols $A_{1}, \cdots, A_{k}$ we have

$F\left(A_{1}, \cdots, A_{k}\right)=G\left(A_{1}, \cdots, A_{k}\right) \rightarrow H\left(A_{1}, \cdots, A_{k}\right)=L\left(A_{1}, \cdots, A_{k}\right)$.

This does not hold reading "R.E.T.s" for "isols" (despite the fact that T3*(b) holds for arbitrary R.E.T.s); take $F\left(A_{1}, A_{2}, A_{3}\right)$ $=\left(A_{1}+1\right) A_{2}, G=\left(A_{1}+1\right) A_{3}, H=A_{2}, L=A_{3}$.

\section{REFERENCES}

1. J. C. E. Dekker, A nonconstructive extension of the number system, J. Symbolic Logic vol. 20 (1955) pp. 204-205.

2. - On certain equations in isols, J. Symbolic Logic vol. 22 (1957) pp. 105106 and Bull. Amer. Math. Soc. vol. 63 (1957) p. 313.

3. J. C. E. Dekker and J. Myhill, Recursive equivalence types, to be published in 1959 by University of California Publications in Mathematics.

4. A. Nerode, Some remarks on isolic arithmetic, Notices Amer. Math. Soc. vol. 6 (1958) p. 216.

5. H. G. Rice, Completely recursively enumerable classes and their key arrays, J. Symbolic Logic vol. 21 (1956) pp. 304-308.

The Institute for Advanced Study. 